\title{
Strategies for the successful implementation of disinfecting port protectors to reduce CLABSI in a large tertiary care teaching hospital
}

Cole Beeler MD a, *, Denise Kerley MSN, RN, CNRN, AG-CNS b, Carmen Davis MSN, RN, CCRN, CNS-BC b, Dana Hazen BSN, RN, MPH c, William Snyderman MPH, CIC c, Kathy Lyons RN, BSN c, Josh Sadowski BS d, Jennifer Sweeney MSN, RN, ACNSBC, CDE d, Lana Dbeibo MD e, Kristen Kelley MPH, RN, BSN, CIC d, Douglas H.

Webb MD, FIDSA, FSHEA $f$

\begin{abstract}
a Division of Infectious Diseases, Indiana University Hospital, Indianapolis, IN b Department of Infection Prevention, Indiana University Hospital, Indianapolis, IN c Department of Infection Prevention, Methodist Hospital, Indianapolis, IN d Department of Infection Prevention, Indiana University Health, Indianapolis, IN e Division of Infectious Diseases, Methodist Hospital, Indianapolis, IN f Division of Infectious Diseases, Indiana University Health, Indianapolis, IN
\end{abstract}

\section{HIGHLIGHTS}

- Disinfecting port protectors can be used to reduce CLABSI.

- Implementation of port protectors into nursing culture can be challenging.

- Evidence-based strategies for implementation can be utilized to sustain their use.

Disinfecting port protectors are a supplement to the central line-associated bloodstream infection prevention bundle as an optional recommendation from the Centers for Disease Control and Prevention. Despite evidence of effectiveness, few centers have successfully reported systematic, sustained implementation of these devices. In this article, we discuss a successful implementation in a large tertiary care teaching hospital, using an evidencebased, multidisciplinary approach.

Key Words: Infection prevention; Bacteremia; Ethanol caps; Bundle measures; Quality improvement; Hub infection

This is the author's manuscript of the article published in final edited form as:

Beeler, C., Kerley, D., Davis, C., Hazen, D., Snyderman, W., Lyons, K., Sadowski, J., Sweeney, J., Dbeibo, L., Kelley, K., \& Webb, D. H. (2019). Strategies for the successful implementation of disinfecting port protectors to reduce CLABSI in a large tertiary care teaching hospital. American Journal of Infection Control, 47(12), $1505-1507$. https://doi.org/10.1016/j.ajic.2019.05.016 
Implementation of technology to reduce central line-associated bloodstream infections (CLABSIs) can help prevent harm by insulating against human error. However, the use of novel devices is not always sustainable owing to a changing workforce, difficulty adapting them to unit workflow, and shifting leadership focus. 1

CLABSI harm reduction strategies include scrubbing of the catheter access port with disinfectant.. Up to $54 \%$ of CLABSI are preceded by port colonization with the same organism. -3 Despite this risk, $56 \%$ of the nurses surveyed responded that they did not feel the need to scrub ports prior to use. ${ }^{4}$ Additionally, complacency with scrub times may lead to inadequate sterilization and infection. $\underline{5}$

Preventative bundle recommendations include the use of alcohol-impregnated disinfecting port protectors (AIDPP) to reduce risk from failure of manual disinfection.? Literature has shown their superiority in bioburden reduction when compared with port scrubbing with disinfectant $\underline{\underline{6}}$ and efficacy in CLABSI reduction. $\underline{\underline{7}}$

Our 1,009-bed tertiary care teaching hospital had previously attempted implementation of the AIDPP to help reduce ongoing high CLABSI rates. Sub-optimal rollout, perceived lack of efficacy by nursing staff, and increased cost to the units led to abandonment of the initiative. In response, a multidisciplinary CLABSI harm team decided to reconsider implementation strategies. This paper describes how our center successfully disseminated and sustained use of the AIDPP for harm reduction evidence-based strategies.

\section{METHODS}

The harm team used the lowa model of evidence-based practice -8 and the 4 tenants of the Cullen and Adams evidence-based practice implementation guide 9 to embed the AIDPP (Curos 3M, St Paul, MN) into the daily nursing workflow (Fig 1). 


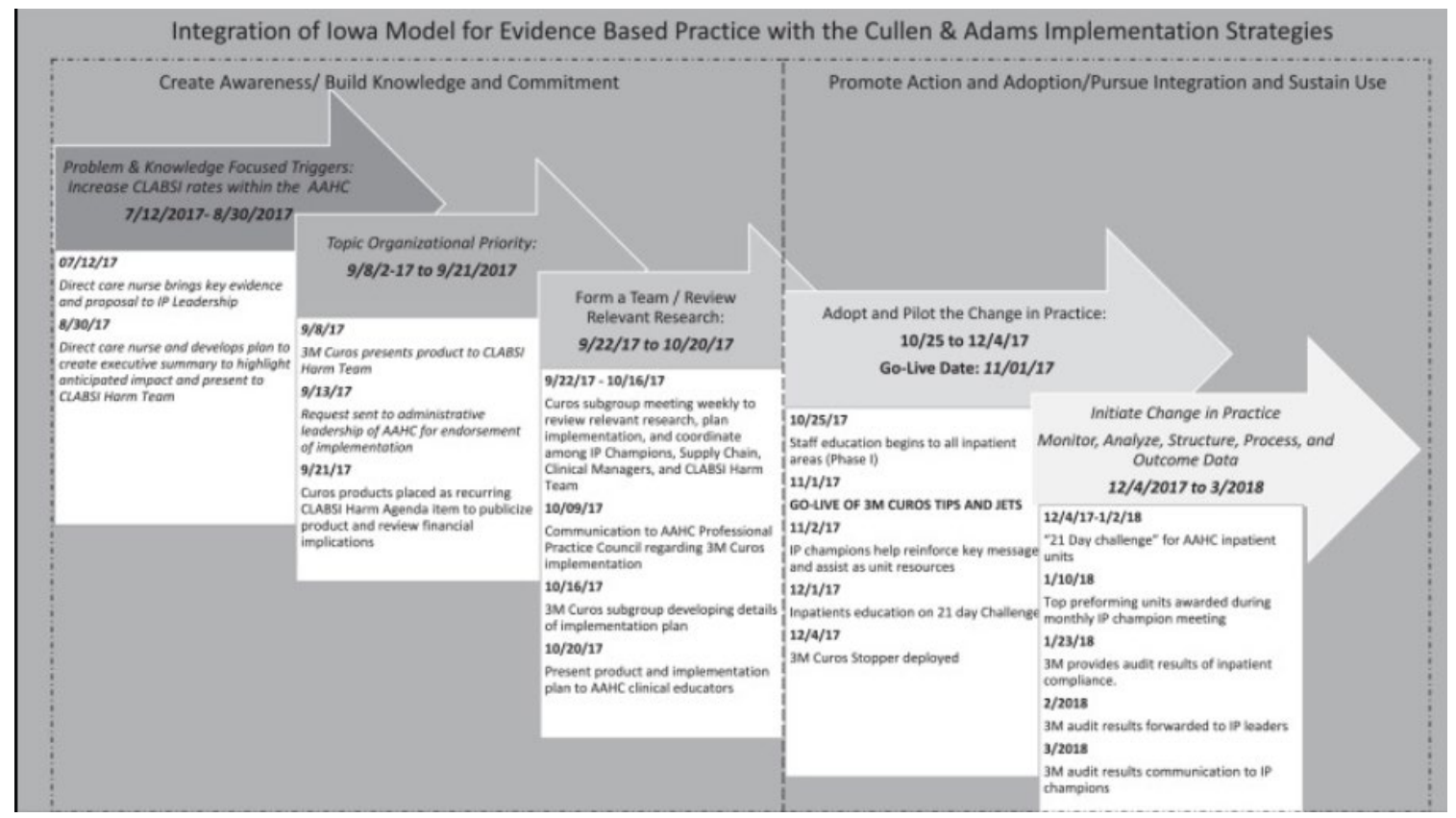

Fig 1. Timeline for implementation of the AIDPP. Arrows depict the lowa model implementation timeline. Dotted lines demonstrate the integration and overlap of each model's systematic approach and successful strategies. AAHC, academic health centers; AIDPP, alcohol-impregnated disinfecting port protectors; CLABSI, central line-associated bloodstream infection.

A high CLABSI rate (Problem-Focused Trigger) prompted leadership to endorse the use of the AIDPP (Topic Organizational Priority). Implementation was assigned to the harm team, given its collaborative position between hospital leadership and the bedside (Form a Team). The harm team gathered evidence and critically appraised the use of the AIDPP on all intravenous access types (central, peripheral, and dialysis catheters), tubing ports and tips, and needleless connectors.

Prior to launch, an executive summary highlighting the key advantages of these devices was distributed to clinicians, organizational leaders, and key stakeholders through existing professional councils. Commitment was fostered using a multifaceted approach that included vendor onsite training, printed and electronic educational materials, unit educator reinforcement, and members of the Infection Prevention Unit Champion Committee (IPUCC). 
The IPUCC was composed of unit-based direct care nurses who have an interest in patient safety. Members developed unit-specific educational strategies and met regularly to discuss barriers, share success stories, and escalate themes to the harm team who could, in turn, escalate to hospital leadership. Figure 2 shows this process created a feedback loop, in which priorities were efficiently communicated between leadership and bedside nursing. Audit results were presented to the IPUCC and shared with bedside nursing to help refine and standardize processes. By rapidly identifying and addressing barriers at the bedside, the harm team was able to foster sustainable adherence (Pilot the Change in Practice).

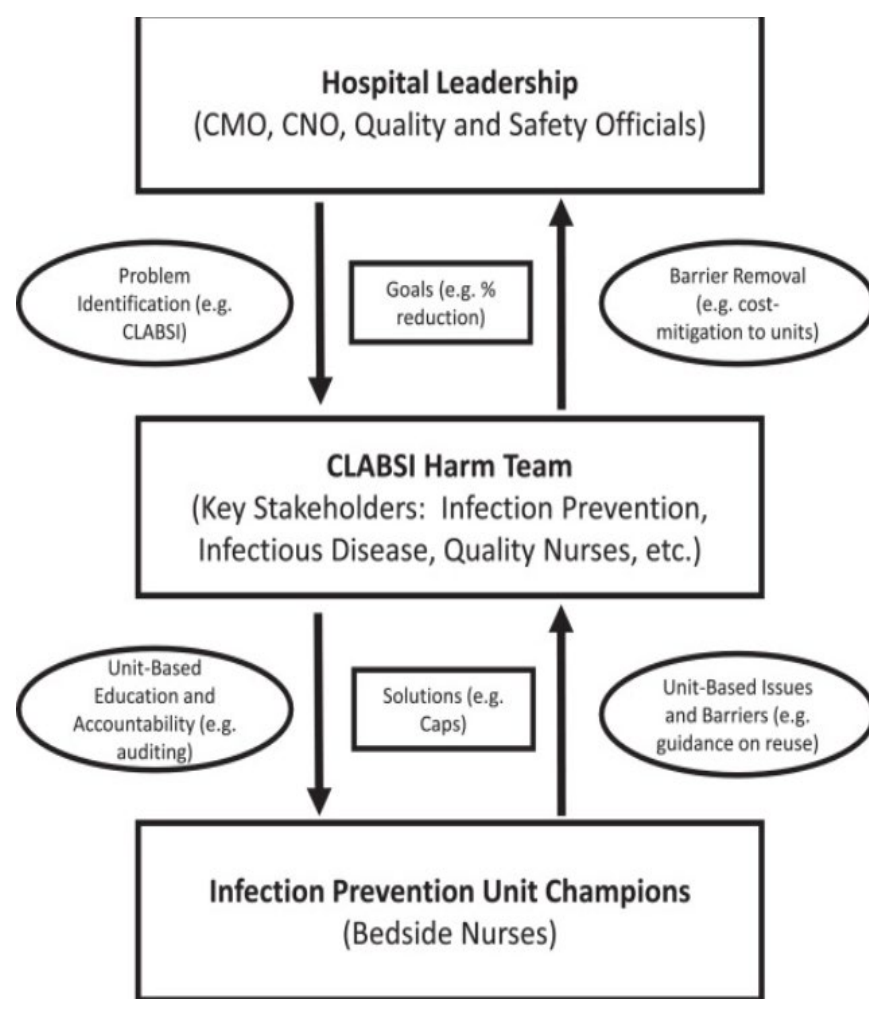

Fig 2. Harm team structure and relationship with the Infection Prevention Unit Champion Committee. CLABSI, central line-associated bloodstream infection; CMO, chief medical officer; $\mathrm{CNO}$, chief nursing officer.

\section{RESULTS}

A point prevalence survey was performed 4 months postimplementation and showed a $67 \%$ adherence rate. In response, the harm team and the IPUCC developed additional unit-specific strategies to increase adherence, including specific education during 
standardized unit orientation. Nine months postimplementation, the AIDPP were added to the CLABSI bundle audit form. Ten months postimplementation, the adherence rate increased to $94 \%$. Total CLABSI bundle compliance for 8 months post-implementation was $81.9 \%$.

In the 8 months pre-implementation, the CLABSI rate was 1.36 infections per 1,000 device days. In the 8 months postimplementation, the rate was sustained at 0.87 . There was no statistically significant difference in the type of lines infected pre- and postimplementation of the AIDPP $(X 2=11.259$, $P$ value $=.13)$. Similarly, organism distribution of CLABSI cases was not significantly different pre- and postimplementation $(\mathrm{X} 2=3.01, \mathrm{P}=.22)$.

The AIDPP cost an average of $\$ 34,000$ per month at our hospital. The average CLABSI costs approximately $\$ 70,696$ per event. 10 Assuming a consistent patient flow and central line utilization rate, our institution had 27 less CLABSI in the 8 months postimplementation, which potentially saved an adjusted $\$ 1,636,792$, accounting for the added cost of the protectors.

\section{DISCUSSION}

The sustainable implementation of the AIDPP reported in this study required a safety culture in which leadership placed a high value on the suggestions of bedside providers. To our knowledge, this is the first project that described this process for the AIDPP using evidence-based strategies. Key evidence and rationale was relayed, from topic experts to bedside nurses, using a systematic dissemination and education plan. Early and regular connection with nursing, which included a competitive 21-day adherence challenge, created a high level of engagement. A structured feedback loop for observations and coaching-in-the-moment by the IPUCC helped to create a standard for this new practice. Last, inclusion of the AIDPP, as a component of the CLABSI bundle, hardwired adherence by audit accountability.

\section{LIMITATIONS}


Because of the multiple simultaneous quality improvement projects that occurred during the implementation period, we cannot state whether the AIDPP were the sole driver of CLABSI reduction. Regardless, the evidence to support the use of these devices was already robust. There was no control group to investigate if other strategies may have been equally viable. Given that this project was implemented within a single center, we cannot confirm that strategies used here would apply to other hospital systems. Despite this, we believe that the philosophies used for implementation are likely universal and can be adopted successfully based on local culture and within pre-existing systems.

\section{CONCLUSIONS}

Implementation of the AIDPP should include a multidisciplinary approach focused on strategic efforts to disseminate education, foster leadership support, and provide consistent feedback to units based on identified trends in adherence.

\section{REFERENCES}

1. McLaws, M.-L., \& Burrell, A. R. (2012). Zero risk for central line-associated bloodstream infection: Are we there yet?*. Critical Care Medicine, 40(2), 388-393.

https://doi.org/10.1097/CCM.0b013e318232e4f3

2. Marschall, J., Mermel, L. A., Fakih, M., Hadaway, L., Kallen, A., O'Grady, N. P., Pettis, A. M., Rupp, M. E., Sandora, T., Maragakis, L. L., \& Yokoe, D. S. (2014). Strategies to Prevent Central Line-Associated Bloodstream Infections in Acute Care Hospitals: 2014 Update. Infection Control \& Hospital Epidemiology, 35(S2), S89-S107.

https://doi.org/10.1017/S0899823X00193870

3. Salzman, M. B., Isenberg, H. D., Shapiro, J. F., Lipsitz, P. J., \& Rubin, L. G. (1993). A Prospective Study of the Catheter Hub as the Portal of Entry for Microorganisms Causing Catheter-Related Sepsis in Neonates. The Journal of Infectious Diseases, 167(2), 487-490. https://doi.org/10.1093/infdis/167.2.487

4. Hadaway, L. (2007). Intermittent Intravenous Administration Sets: Survey of Current Practices. Journal of the Association for Vascular Access, 12(3), 143-147. https://doi.org/10.2309/java.12-3-10 
5. Kaler, W., \& Chinn, R. (2007). Successful Disinfection of Needleless Access Ports: A Matter of Time and Friction. Journal of the Association for Vascular Access, 12(3), 140142. https://doi.org/10.2309/java.12-3-9

6. DeVries, M., Mancos, P. S., \& Valentine, M. J. (2014). Reducing Bloodstream Infection Risk in Central and Peripheral Intravenous Lines: Initial Data on Passive Intravenous Connector Disinfection. Journal of the Association for Vascular Access, 19(2), 87-93. https://doi.org/10.1016/j.java.2014.02.002

7. Merrill, K. C., Sumner, S., Linford, L., Taylor, C., \& Macintosh, C. (2014). Impact of universal disinfectant cap implementation on central line-associated bloodstream infections. American Journal of Infection Control, 42(12), 1274-1277. https://doi.org/10.1016/j.ajic.2014.09.008

8. Buckwalter, K. C., Cullen, L., Hanrahan, K., Kleiber, C., McCarthy, A. M., Rakel, B., Steelman, V., Tripp-Reimer, T., \& Tucker, S. (2017). lowa Model of Evidence-Based Practice: Revisions and Validation. Worldviews on Evidence-Based Nursing, 14(3), 175-182. https://doi.org/10.1111/wvn.12223

9. Cullen, L., \& Adams, S. L. (2012). Planning for Implementation of Evidence-Based Practice. JONA: The Journal of Nursing Administration, 42(4), 222-230.

\section{https://doi.org/10.1097/NNA.0b013e31824ccd0a}

10. Berenholtz, S. M., Lubomski, L. H., Weeks, K., Goeschel, C. A., Marsteller, J. A., Pham, J. C., Sawyer, M. D., Thompson, D. A., Winters, B. D., Cosgrove, S. E., Yang, T., Louis, T. A., Lucas, B. M., George, C. T., Watson, S. R., Albert-Lesher, M. I., Andre, J. R. S., Combes, J. R., Bohr, D., ... Class='sup'>a</Span>, on behalf of the O. the C. S. B. program<span. (2014). Eliminating Central Line-Associated Bloodstream Infections: A National Patient Safety Imperative. Infection Control \& Hospital Epidemiology, 35(1), 56-62. https://doi.org/10.1086/674384 